\title{
Erratum to: Asymptotic behaviour of first passage time distributions for Lévy processes
}

\author{
R. A. Doney ${ }^{1} \cdot$ V. Rivero ${ }^{2}$
}

Published online: 30 September 2015

(C) Springer-Verlag Berlin Heidelberg 2015

\section{Erratum to: Probab Theory Relat Fields (2013) 157:1-45 DOI 10.1007/s00440-012-0448-x}

In this Erratum, we correct an error in our paper "Asymptotic behaviour of first passage time distributions for Lévy processes" published in Probab Theory Relat Fields, 157(1-2):1-45, 2013.

Let $X$ be a real valued Lévy process with law $\mathbb{P}$, characteristic exponent $\Psi$ and characteristic triplet $(a, \sigma, \Pi)$. We assume that $X$ is in the domain of attraction of a stable distribution without centering, that is there exists a deterministic function $c:(0, \infty) \rightarrow(0, \infty)$ such that

$$
\frac{X_{t}}{c(t)} \stackrel{\mathcal{D}}{\rightarrow} Y_{1}, \quad \text { as } t \rightarrow \infty,
$$

with $Y_{1}$ a strictly stable random variable of parameter $0<\alpha \leq 2$, and positivity parameter $\rho=\mathbb{P}\left(Y_{1}>0\right)$. In this case we will use the notation $X \in D(\alpha, \rho)$, and put $\bar{\rho}=1-\rho$. Hereafter $\left(Y_{t}, t \geq 0\right)$ will denote an $\alpha$-stable Lévy process with positivity parameter $\rho=\mathbb{P}\left(Y_{1}>0\right)$. We write $f$ for the density of $Y_{1}$, and $\Psi_{\alpha}$ for its characteristic exponent.

The online version of the original article can be found under doi:10.1007/s00440-012-0448-x.

\footnotetext{
$\triangle \quad$ V. Rivero

rivero@cimat.mx

R. A. Doney

ron.doney@manchester.ac.uk

1 Department of Mathematics, University of Manchester, Manchester M13 9PL, UK

2 Centro de Investigacion en Matematicas A.C., Calle Jalisco s/n, 36240 Guanajuato, Mexico
} 
In the original paper we provided sharp estimates for the local behaviour of the distribution of the first passage time of $X$ below 0 , i.e. $T_{0}=\inf \left\{t>0: X_{t}<0\right\}$, under $\mathbb{P}_{x}(\cdot)$, for $x>0$, both in the event of creeping and non-creeping. The proof of our results has been based in the validity of the following result, Proposition 13 in the original paper.

Proposition 1 Assume that $X \in D(\alpha, \rho)$. Then uniformly in $0<\Delta<\Delta_{0}$, with $\Delta_{0}>0$, and $x \in \mathbb{R}$,

$$
c(t) \mathbb{P}\left(X_{t} \in(x, x+\Delta]\right)=\Delta\left(f\left(\frac{x}{c(t)}\right)+o(1)\right) \text { as } t \rightarrow \infty .
$$

Consequently, given any $\Delta_{0}>0$ there are constants $k_{0}$ and $t_{0}$ such that

$$
c(t) \mathbb{P}\left(X_{t} \in(x, x+\Delta]\right) \leq k_{0} \Delta, \quad \text { for all } t \geq t_{0} \text { and } \Delta \in\left(0, \Delta_{0}\right]
$$

We claimed that this result can easily be proved by repeating the argument used for non-lattice random walks in [3], with very minor changes. This fact is indeed true to some extent, but the uniformity in $\Delta$ is only true in general on intervals $[a, b]$ with $0<a<b<\infty$; and further assumptions are needed to obtain the uniformity on $0<\Delta<\Delta_{0}$, for $\Delta_{0}>0$. Hence for our results in original paper to be valid we require two extra assumptions, namely $(\mathrm{H} 2)$ and $(\mathrm{H} 3)$ below.

(H1) $X \in D(\alpha, \rho)$.

(H2) There exists a $t_{0}>0$ such that

$$
\int_{|\lambda|>1} e^{-t_{0} \Re \Psi(\lambda)} d \lambda<\infty
$$

(H3) $X$ is strongly non-lattice,

$$
m=\liminf _{|\lambda| \rightarrow \infty} \Re \Psi(\lambda)>0 .
$$

Observe that the assumption (H2) implies that the law of $X_{t}$ has a density for all $t>t_{0}$, see e.g. Proposition 2.5 in [2].

Under these assumptions we have the following Lemma which replaces the Proposition 13 in the original paper.

Lemma 2 Assume (H1-3) hold. We have that

$$
c(t) \mathbb{P}\left(X_{t} \in(x, x+\Delta]\right)=\Delta\left(f\left(\frac{x}{c(t)}\right)+o(1)\right), \text { as } t \rightarrow \infty,
$$

uniformly in $x \in \mathbb{R}$, and $0 \leq \Delta<\Delta_{0}$. Consequently (3) holds.

Proof We would like to estimate

$$
c(t) \mathbb{P}\left(X_{t} \in(x, x+\Delta]\right)
$$


uniformly in $x \in \mathbb{R}$, and uniformly in $|\Delta|<\Delta_{0}$ for $\Delta_{0}$ fixed. For $\Delta>0$, the function

$$
g_{\Delta, t}(x)=\frac{1}{\Delta} \mathbb{P}\left(X_{t} \in(x, x+\Delta]\right), \quad x \in \mathbb{R},
$$

is a probability density function, that of $X_{t}+U_{\Delta}$, with $U_{\Delta}$ an independent r.v. with uniform distribution over $(-\Delta, 0)$. Its characteristic function is given by

$$
\widehat{g}_{\Delta, t}(\lambda):=\int_{\mathbb{R}} e^{i \lambda x} g_{\Delta, t}(x) d x=\mathbb{E}\left(e^{i \lambda X_{t}}\right) \frac{\left(1-e^{-i \lambda \Delta}\right)}{i \lambda \Delta}=e^{-t \Psi(\lambda)} \frac{\left(1-e^{-i \lambda \Delta}\right)}{i \lambda \Delta}
$$

The integrability assumption (H2) implies that for $t>t_{0}$, and $\Delta>0$

$$
\int_{-\infty}^{\infty}\left|\widehat{g}_{\Delta, t}(\lambda)\right| d \lambda<\infty
$$

By the Fourier inversion theorem we have that for $\Delta>0, t>t_{0}$

$$
\begin{aligned}
& \Delta c(t) g_{\Delta, t}(x)-\Delta f(x / c(t)) \\
& =\frac{\Delta}{2 \pi} \int_{-\infty}^{\infty} d \lambda e^{-i x \lambda / c(t)}\left[e^{-t \Psi(\lambda / c(t))}-e^{-\Psi_{\alpha}(\lambda)}\right]\left(\frac{1-e^{-i \lambda \Delta / c(t)}}{i \lambda \Delta / c(t)}\right) \\
& \quad+\frac{\Delta}{2 \pi} \int_{-\infty}^{\infty} d \lambda e^{-i x \lambda / c(t)} e^{-\Psi_{\alpha}(\lambda)}\left(\frac{1-e^{-i \lambda \Delta / c(t)}}{i \lambda \Delta / c(t)}-1\right) .
\end{aligned}
$$

To estimate this expression we will use among other things the inequalities

$$
\left|\frac{e^{i u}-1}{i u}\right| \leq 1, \quad\left|\frac{e^{i u}-1-i u}{i u}\right| \leq \frac{|u|}{2}, \quad u \in \mathbb{R}
$$

see [2] Lemma 8.6. Using the latter, the second term in the above expression can be estimated by

$$
\begin{aligned}
& \frac{\Delta}{2 \pi}\left|\int_{-\infty}^{\infty} d \lambda e^{-i x \lambda / c(t)} e^{-\Psi_{\alpha}(\lambda)}\left(\frac{1-e^{-i \lambda \Delta / c(t)}}{i \lambda \Delta / c(t)}-1\right)\right| \\
& \leq \frac{\Delta}{4 \pi}\left(\frac{\Delta_{0}}{c(t)}\right) \int_{-\infty}^{\infty} d \lambda e^{-\Re \Psi_{\alpha}(\lambda)}|\lambda| .
\end{aligned}
$$

Since $\Re \Psi_{\alpha}(\lambda)=|\lambda|^{\alpha} c_{\alpha}$, with $c_{\alpha} \in(0, \infty)$ a constant, the latter integral is finite, and hence its product with $\Delta / c(t)$ tends to zero uniformly in $x$ and $\Delta$, as long as $\Delta$ remains bounded.

Because $X \in D(\alpha, \rho)$ we have that

$$
\lim _{t \rightarrow \infty}\left|\exp \{-t \Psi(\lambda / c(t))\}-e^{-\Psi_{\alpha}(\lambda)}\right|=0,
$$


uniformly over closed intervals $[-A, A]$, and also $\left|\frac{1-e^{-i \lambda \Delta / c(t)}}{i \lambda \Delta / c(t)}\right| \leq 1$. It follows that for any $A>0$

$$
\Delta\left|\int_{-A}^{A} d \lambda e^{-i x \lambda / c(t)}\left[e^{-t \Psi(\lambda / c(t))}-e^{-\Psi_{\alpha}(\lambda)}\right]\left(\frac{1-e^{-i \lambda \Delta / c(t)}}{i \lambda \Delta / c(t)}\right)\right| \rightarrow 0, \quad t \rightarrow \infty
$$

uniformly in $x$ and $\Delta<\Delta_{0}$. To finish it will be sufficient to prove that

$$
\Delta \int_{(-A, A)^{c}} d \lambda e^{-t \Re \Psi(\lambda / c(t))} \rightarrow 0,
$$

uniformly in $x$, and $\Delta<\Delta_{0}$. We proceed as follows. Because the function $\lambda \mapsto$ $\Re \Psi(\lambda)$ is regularly varying at 0 , the Potter bounds, [1] Theorem 1.5.6, ensure that for any $\alpha>\epsilon>0$ there exists constant $K$ and a $B_{1}$ such that

$$
\Re \Psi(\lambda) \geq K \lambda^{\alpha-\epsilon}, \quad 0 \leq \lambda<B_{1}
$$

We apply this inequality to infer

$$
\Delta \int_{A}^{B_{1} c(t)} d \lambda e^{-t \Re \Psi(\lambda / c(t))} \leq \Delta \int_{0}^{\infty} d \lambda e^{-K t\left(\frac{\lambda}{c(t)}\right)^{\alpha-\epsilon}}
$$

An application of the monotone convergence theorem shows that the latter term tends to 0 , as $t \rightarrow \infty$, because $c(t) \in R V_{1 / \alpha}^{\infty}$ and therefore $t /(c(t))^{\alpha-\epsilon} \in R V_{\epsilon / \alpha}^{\infty}$. The convergence is uniform in $x$, and in $\Delta$ on bounded intervals. By symmetry we also get the convergence

$$
\Delta \int_{-B_{1} c(t)}^{-A} d \lambda e^{-t \Re \Psi(\lambda / c(t))} \underset{t \rightarrow \infty}{\longrightarrow} 0
$$

uniformly in $x$, and in $\Delta$ on bounded intervals. The assumption of having $X$ strongly non-lattice, implies that, given $\epsilon>0$ and small enough, there is a $B_{2}$ such that $\Re \Psi(\lambda)>m-\epsilon>0$ for all $|\lambda|>B_{2}$. By the continuity of $\Re \Psi(\lambda)$ and the fact that this function does not take the value zero in $\mathbb{R} \backslash\{0\}$, since $X$ is non-lattice, we can assume that $B_{2}=B_{1}$, maybe at the price of replacing $m-\epsilon$ by $0<\widetilde{m} \leq m-\epsilon$. We get that for $t>t_{0}>0$

$$
\begin{aligned}
\Delta \int_{\left(B_{2} c(t), \infty\right)} d \lambda e^{-t \Re \Psi(\lambda / c(t))} & \leq \Delta c(t) \int_{B_{2}}^{\infty} \exp \{-t \Re \Psi(\lambda)\} d \lambda \\
& \leq \Delta c(t) \exp \left\{-\left(t-t_{0}\right) \widetilde{m}\right\} \int_{B_{2}}^{\infty} \exp \left\{-t_{0} \Re \Psi(\lambda)\right\} d \lambda .
\end{aligned}
$$

The rightmost term in the above inequality tends to zero as $t \rightarrow \infty$, because the function $c(\cdot)$ is regularly varying and hence its growth is at most polynomial. By symmetry we deduce the convergence 


$$
\Delta \int_{\left(-\infty,-B_{2} c(t)\right)} d \lambda e^{-t \Re \Psi(\lambda / c(t))} \underset{t \rightarrow \infty}{\longrightarrow} 0
$$

uniformly in $x$, and in $\Delta$ on bounded intervals. The result follows.

\section{References}

1. Bingham, N.H., Goldie, C.M., Teugels, J.L.: Regular variation. Encyclopedia of Mathematics and Its Applications, vol. 27. Cambridge University Press, Cambridge (1989)

2. Sato, K.: Lévy processes and infinitely divisible distributions. In: Cambridge Studies in Advanced Mathematics, vol. 68. Cambridge University Press, Cambridge (2013)

3. Stone, C.: On local and ratio limit theorems. In: Proceedings of the 5th Berkeley Sympos. Math. Statist. and Probability (Berkeley, Calif., 1965/66), vol. II. Contributions to Probability Theory, Part 2, pp. 217-224. University of California Press, Berkeley (1967) 